\title{
Information Complexity and Generalization Bounds
}

\author{
Pradeep Kr. Banerjee* Guido Montúfar ${ }^{\dagger}$
}

\begin{abstract}
We present a unifying picture of PAC-Bayesian and mutual information-based upper bounds on the generalization error of randomized learning algorithms. As we show, Tong Zhang's information exponential inequality (IEI) gives a general recipe for constructing bounds of both flavors. We show that several important results in the literature can be obtained as simple corollaries of the IEI under different assumptions on the loss function. Moreover, we obtain new bounds for data-dependent priors and unbounded loss functions. Optimizing the bounds gives rise to variants of the Gibbs algorithm, for which we discuss two practical examples for learning with neural networks, namely, Entropy- and PAC-Bayes- SGD. Further, we use an Occam factor argument to show a PAC-Bayesian bound that incorporates second-order curvature information of the training loss.
\end{abstract}

\section{Contents}

1 Introduction $\quad 2$

2 Preliminaries $\quad 3$

3 One bound to rule them all $\quad 4$

3.1 The information exponential inequality . . . . . . . . . . . . . . 4

3.2 The conditional mutual information $(\mathrm{CMI})$ bound $\ldots \ldots \ldots \ldots$

3.3 Recovering classical PAC-Bayesian bounds . . . . . . . . . . . . . . 8

4 Differentially private data-dependent priors $\quad 9$

5 Information complexity minimization $\quad 10$

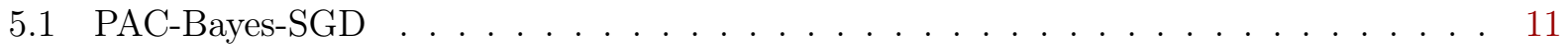

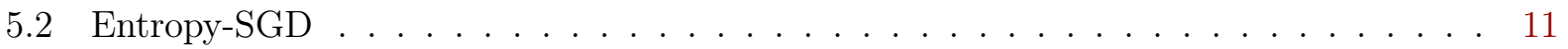

5.3 PAC-Bayes and Occam factor . . . . . . . . . . . . . . . . . 12

6 Discussion $\quad 12$

A The strong functional representation lemma and single-draw bounds

B Proofs

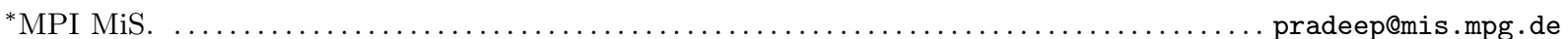

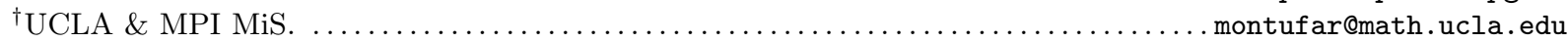




\section{Introduction}

The generalization capability of a learning algorithm is intrinsically related to the information that the output hypothesis reveals about the input training dataset: The lesser the information revealed, the better the generalization. This argument has been formalized in recent years by appealing to different notions of information stability [1-9]. Information stability quantifies the sensitivity of a learning algorithm to local perturbations of its input, and draws on a rich tradition of earlier work on algorithmic [10-12], and distributional [13-15] stability in adaptive data analysis. Closely related to the information stability approach is the so-called PAC-Bayesian approach to data-dependent generalization bounds, originally due to McAllester [16-18]. While these two approaches have evolved independently of each other, a principal objective of this work is to present them under a unified framework.

We consider the standard apparatus of statistical learning theory [19]. We have an example domain $\mathcal{Z}=\mathcal{X} \times \mathcal{Y}$ of the instances and labels, a hypothesis space $\mathcal{W}$, a fixed loss function $\ell$ : $\mathcal{W} \times \mathcal{Z} \rightarrow[0, \infty)$, and a training sample $S$, which is an $n$-tuple $\left(Z_{1}, \ldots, Z_{n}\right)$ of i.i.d. random elements of $\mathcal{Z}$ drawn according to some unknown distribution $\mu$. A learning algorithm is a Markov kernel $P_{W \mid S}$ that maps input training samples $S$ to conditional distributions of hypotheses $W$ in $\mathcal{W}$. This defines a joint distribution $P_{S W}=P_{S} P_{W \mid S}, P_{S}=\mu^{\otimes n}$, and a corresponding marginal distribution $P_{W}$. The true risk of a hypothesis $w \in \mathcal{W}$ on $\mu$ is $L_{\mu}(w):=\mathbb{E}_{\mu}[\ell(w, Z)]$, and its empirical risk on the training sample $S$ is $L_{S}(w):=\frac{1}{n} \sum_{i=1}^{n} \ell\left(w, Z_{i}\right)$. Our goal is to control the generalization error, $\mathrm{g}(W, S):=L_{\mu}(W)-L_{S}(W)$, either in expectation, or with high probability. One difficulty in achieving this goal is the nontrivial statistical dependency between the sample $S$ and the learned hypothesis $W$.

For controlling the generalization error in expectation, we can rewrite the true risk of a given hypothesis $w$ as $L_{\mu}(w)=\mathbb{E}_{S^{\prime} \sim \mu^{\otimes n}}\left[L_{S^{\prime}}(w)\right]$, where $S^{\prime}=\left(Z_{1}^{\prime}, \ldots, Z_{n}^{\prime}\right)$ is an i.i.d. sample. Then the expected generalization error can be written as a difference of two expectations of the same loss function,

$$
\mathbb{E}_{S W}[\mathrm{~g}(W, S)]=\mathbb{E}_{P_{S} \otimes P_{W}}\left[L_{S}(W)\right]-\mathbb{E}_{P_{S W}}\left[L_{S}(W)\right],
$$

where the second expectation is taken w.r.t. the joint distribution of the training sample and the output hypothesis, while the first expectation is taken w.r.t. the product of the two marginal distributions. Hence the expected generalization error reflects the dependence of the output $W$ on the input $S$. This dependence can also be measured by their mutual information as has been shown in recent works [2-7]. We refer to such bounds as mutual information-based generalization bounds.

Alternatively, we may wish to control the generalization error of the learning algorithm $P_{W \mid S}$ with high probability over the training sample $S$. The expected generalization error over hypotheses chosen from the distribution $P$ (posterior) output by the learning algorithm, i.e., $\mathbb{E}_{P}[\mathrm{~g}(W, S)]$, can be upper-bounded with high probability under $P_{S}$ by the KL divergence between $P$ and an arbitrary reference distribution $Q$ (prior), that is selected before the draw of the training sample $S$. For any $Q$, these bounds hold uniformly for all $P$, and are called PAC-Bayesian bounds [16-18,20-29], where PAC stands for Probably Approximately Correct. Bounds of this type are useful when we have a fixed dataset $s \in \mathcal{Z}^{n}$ and a new hypothesis is sampled from $P$ every time the algorithm is used. Choosing the posterior to minimize a PAC-Bayesian bound leads to the well-known Gibbs-ERM principle $[2,20,25,30]$. On the other hand, for a fixed posterior $P, \mathbb{E}_{S}[D(P \| Q)]$ is minimized by the oracle prior, $Q^{\star}=\mathbb{E}_{S}\left[P_{W \mid S}(\cdot \mid S)\right]$. Note $\mathbb{E}_{S}\left[D\left(P \| Q^{\star}\right)\right]$ is just the mutual information $I(S ; W)$, which is the key quantity controlling the expected generalization error in [2-4]. 
Summary of contributions. We present a unified framework for deriving PAC-Bayesian and mutual information-based generalization bounds, starting from a fundamental information-theoretic inequality, Lemma 4, due to Tong Zhang [20]. Besides recovering several well-known bounds of both flavors, such as the Xu-Raginsky mutual information-bound [2] in Corollary 7, and Catoni's bound [21] in Corollary 13, we also obtain new bounds for data-dependent priors (Proposition 19) and unbounded loss functions (Theorem 6 and Proposition 10). Proposition 12 gives a PAC-Bayes version of the CMI bound due to Steinke and Zakynthinou [7]. Optimizing these bounds w.r.t. the posterior gives rise to variants of the Gibbs algorithm, for which we discuss two examples and show how Catoni's bound can be used to derive a PAC-Bayes-SGD [31] objective. In Proposition 22, we give a PAC-Bayesian bound motivated by an Occam's factor argument, in relation to "flat" minima in neural networks [32].

\section{Preliminaries}

We write $\mathcal{M}(\mathcal{W})$ to denote the family of probability measures over a set $\mathcal{W}$, and $\mathcal{K}(\mathcal{S}, \mathcal{W})$ to denote the set of Markov kernels from $\mathcal{S}$ to $\mathcal{W}$. Proposition 1 collects some well-known facts about the cumulant generating function $\Lambda_{X}(\beta)=\ln \mathbb{E}\left[e^{\beta X}\right]$ of a random variable $X$ for $\beta>0$ (see, e.g., [33, §2], and [20]):

Proposition 1 (Facts about the cumulant generating function $\Lambda_{X}(\beta)$ for $\beta>0$ ).

1. $\Lambda_{X}(\beta)$ is infinitely differentiable and convex in $\beta$;

2. $\frac{1}{\beta} \Lambda_{X}(\beta)$ is an increasing function of $\beta$;

3. $\mathbb{E}[X] \leq \frac{1}{\beta} \Lambda_{X}(\beta) \leq \Lambda_{X}^{\prime}(\beta)$;

4. For real constants $a, b, \frac{1}{\beta} \Lambda_{a X+b}(\beta)=\frac{1}{\beta} \Lambda_{X}(a \beta)+b$;

5. $\frac{1}{\beta} \Lambda_{X}(\beta) \leq \mathbb{E}[X]+\frac{\beta}{2} \operatorname{Var}(X)+O\left(\beta^{2}\right)$, where $\operatorname{Var}(X)=\mathbb{E}\left[(X-\mathbb{E}[X])^{2}\right] ;$

6. If $X \in[0,1]$, then $\frac{1}{\beta} \Lambda_{X}(\beta) \leq \frac{1}{\beta} \ln \left(1-\left(1-e^{\beta}\right) \mathbb{E}[X]\right)$, with equality when $X \in\{0,1\}$ is Bernoulli;

7. $X$ is $\sigma$-sub-Gaussian if $\frac{1}{\beta} \Lambda_{X}(\beta) \leq \mathbb{E}[X]+\frac{\beta \sigma^{2}}{2}$;

8. $X$ is $(\sigma, c)$-sub-gamma if $\frac{1}{\beta} \Lambda_{X}(\beta) \leq \mathbb{E}[X]+\frac{\beta \sigma^{2}}{2(1-c \beta)}$ for every $\beta$ such that $\beta \in\left(0, \frac{1}{c}\right)$.

We also note that $\Lambda_{X}(0)=0, \Lambda_{X}^{\prime}(0)=\mathbb{E}[X]$.

We will need the following characterization of the inverse of the Fenchel-Legendre dual of a smooth convex function:

Lemma 2 ([33, Lemma 2.4]). Let $\psi$ be a convex and continuously differentiable function defined on the interval $[0, b)$, where $0<b \leq \infty$. Assume that $\psi(0)=\psi^{\prime}(0)=0$. Then, the Legendre dual of $\psi$, defined as

$$
\psi^{*}(t):=\sup _{\beta \in[0, b)}\{\beta t-\psi(\beta)\}
$$


is a nonnegative convex and nondecreasing function on $[0, \infty)$ with $\psi^{*}(0)=0$. Moreover, for every $y \geq 0$, the set $\left\{t \geq 0: \psi^{*}(t)>y\right\}$ is non-empty and the generalized inverse of $\psi^{*}$ defined by $\psi^{*-1}(y)=\inf \left\{t \geq 0: \psi^{*}(t)>y\right\}$ can also be written as

$$
\psi^{*-1}(y)=\inf _{\beta \in(0, b)} \frac{y+\psi(\beta)}{\beta} .
$$

We will need the following property of the Gibbs measure:

Lemma 3 ([20, Proposition 3.1]). For any real-valued measurable function $f$ on $\mathcal{W}$, any real $\beta>0$, and any $P, Q \in \mathcal{M}(\mathcal{W})$ such that $D(P \| Q)<\infty$, we have

$$
\beta^{-1} D\left(P \| P^{*}\right)=\mathbb{E}_{P}[f(W)]+\beta^{-1} D(P \| Q)+\beta^{-1} \ln \mathbb{E}_{Q}\left[e^{-\beta f(W)}\right],
$$

where $P^{*}$ is the Gibbs measure

$$
P^{*}(\mathrm{~d} w):=\frac{e^{-\beta f(w)}}{\mathbb{E}_{Q}\left[e^{-\beta f\left(W^{\prime}\right)}\right]} Q(\mathrm{~d} w) .
$$

Consequently,

$$
\inf _{P \in \mathcal{M}(\mathcal{W})}\left\{\mathbb{E}_{P} f(W)+\beta^{-1} D(P \| Q)\right\}=-\beta^{-1} \ln \mathbb{E}_{Q}\left[e^{-\beta f(W)}\right] .
$$

Finally, we recall the golden formula: For all $Q \in \mathcal{M}(\mathcal{W})$ such that $D\left(P_{W} \| Q\right)<\infty$, we have

$$
I(S ; W)=D\left(P_{W \mid S} \| Q \mid P_{S}\right)-D\left(P_{W} \| Q\right),
$$

where $D\left(P_{W \mid S} \| Q \mid P_{S}\right)=\int_{\mathcal{Z}^{n}} D\left(P_{W \mid S=s} \| Q\right) \mu^{\otimes n}(\mathrm{~d} s)$.

All information-theoretic quantities are expressed in nats, unless specified otherwise. All proofs are relegated to Appendix B.

\section{One bound to rule them all}

\subsection{The information exponential inequality}

For any real $\beta>0$, define

$$
M_{\beta}(w)=-\beta^{-1} \Lambda_{-\ell(w, Z)}(\beta)=-\beta^{-1} \ln \mathbb{E}_{\mu}\left[e^{-\beta \ell(w, Z)}\right],
$$

which acts as a surrogate for $L_{\mu}(w)$. Following [24], we call this quantity the annealed expectation.

Lemma 4 (Information exponential inequality (IEI) [20, Lemma 2.1]). For any prior $Q \in \mathcal{M}(\mathcal{W})$, any real-valued loss function $\ell$ on $\mathcal{W} \times \mathcal{Z}$, and any posterior distribution $P \ll Q$ over $\mathcal{W}$ that depends on an i.i.d. training sample $S$, we have

$$
\mathbb{E}_{S} \exp \left\{n \beta \mathbb{E}_{P}\left[M_{\beta}(W)-L_{S}(W)\right]-D(P \| Q)\right\} \leq 1 .
$$

The IEI implies bounds both in probability and in expectation for the quantity

$$
n \beta \mathbb{E}_{P}\left[M_{\beta}(W)-L_{S}(W)\right]-D(P \| Q),
$$

and is the key tool for showing the following theorem due to Tong Zhang that holds for unbounded loss functions: 
Theorem 5 ([20, Theorem 2.1]). Let $\mu$ be a distribution over $\mathcal{Z}$, and let $S$ be an i.i.d. training sample from $\mu$. Let $Q \in \mathcal{M}(\mathcal{W})$ be a prior distribution that does not depend on $S$, and let $\ell$ be a real-valued loss function on $\mathcal{W} \times \mathcal{Z}$. Let $\beta>0$, and let $\delta \in(0,1]$. Then, with probability of at least $1-\delta$ over the choice of $S \sim \mu^{\otimes n}$, for all distributions $P \ll Q$ over $\mathcal{W}$ (even such that depend on $S)$, we have:

$$
\mathbb{E}_{P}\left[M_{\beta}(W)\right] \leq \mathbb{E}_{P}\left[L_{S}(W)\right]+\frac{1}{n \beta}\left(D(P \| Q)+\ln \frac{1}{\delta}\right)
$$

Moreover, we have the following bound in expectation:

$$
\mathbb{E}_{S W}\left[M_{\beta}(W)\right] \leq \mathbb{E}_{S W}\left[L_{S}(W)\right]+\frac{1}{n \beta} D\left(P \| Q \mid P_{S}\right)
$$

Following [34], we call the regularized empirical risk

$$
\mathbb{E}_{P}\left[L_{S}(W)\right]+\frac{1}{n \beta} D(P \| Q)
$$

as the Information Complexity (IC), which is a data- and algorithm- dependent quantity.

It is useful to replace the annealed expectation $M_{\beta}(w)$ in (3) and (4) with the true risk $L_{\mu}(w)$. By Proposition 1 items 1) and 3), we have $M_{\beta}(w) \leq L_{\mu}(w)$. For general loss functions, Proposition 1 item 5) is useful for getting bounds in the opposite direction. By items 4), 7) and 8) of Proposition 1 , if for all $w \in \mathcal{W}, \ell(w, Z)$ is $\sigma$-sub-Gaussian, resp., $(\sigma, c)$-sub-gamma under $\mu$, then we have for all $w \in \mathcal{W}, L_{\mu}(w) \leq M_{\beta}(w)+\frac{\beta}{2} \sigma^{2}$ for all $\beta>0$, resp., $L_{\mu}(w) \leq M_{\beta}(w)+\frac{\beta}{2(1-c \beta)} \sigma^{2}$ for every $\beta$ such that $\beta \in\left(0, \frac{1}{c}\right)$. More generally, we note the following result, which follows as a corollary to Theorem 5 and Lemma 2:

Theorem 6. Suppose that there exist a convex function $\psi: \mathbb{R}_{\geq 0} \rightarrow \mathbb{R}$ satisfying $\psi(0)=\psi^{\prime}(0)=0$, such that

$$
\sup _{w \in \mathcal{W}}\left[L_{\mu}(w)-M_{\beta}(w)\right] \leq \frac{\psi(\beta)}{\beta}, \forall \beta>0
$$

Then, under the setting of Theorem 5, with probability of at least $1-\delta$ over the choice of $S \sim \mu^{\otimes n}$, for all distributions $P \ll Q$ over $\mathcal{W}$ (even such that depend on $S$ ), we have

$$
\mathbb{E}_{P}[\mathrm{~g}(W, S)] \leq \frac{1}{n \beta}\left(D(P \| Q)+\ln \frac{1}{\delta}\right)+\frac{\psi(\beta)}{\beta} .
$$

Moreover, we have the following bound in expectation:

$$
\mathbb{E}_{S W}[\mathrm{~g}(W, S)] \leq \psi^{*-1}\left(\frac{D\left(P \| Q \mid P_{S}\right)}{n}\right) .
$$

By the golden formula (1), under the oracle prior $Q^{\star}=\mathbb{E}_{S}\left[P_{W \mid S}\right], \mathbb{E}_{S}\left[D\left(P \| Q^{\star}\right)\right]=I(S ; W)$. If $\ell(w, Z)$ is $\sigma$-sub-Gaussian under $\mu$ for all $w \in \mathcal{W}$, then we can take $\psi(\beta)=\frac{\beta^{2} \sigma^{2}}{2}$ for every $\beta>0$ and $\psi^{*-1}(y)=\sqrt{2 \sigma^{2} y}[33, \S 2.3]$, in which case we recover the bound in expectation due to Xu and Raginsky [2]: 
Corollary 7. If $\ell(w, Z)$ is $\sigma$-sub-Gaussian under $\mu$ for all $w \in \mathcal{W}$, then

$$
\mathbb{E}_{S W}[\mathrm{~g}(W, S)] \leq \sqrt{2 \sigma^{2} I(S ; W) / n} .
$$

Corollary 7 shows that an algorithm that reveals a small amount of information about its input generalizes well. This observation, for instance, forms the basis for the Gibbs algorithm, which can be thought of as "stabilizing" the empirical risk minimization (ERM) algorithm by controlling the input-output mutual information $I(S ; W)$ [2]. We discuss extensions of this idea in Section 5. In Appendix A, we highlight a functional characterization of the mutual information in relation to the "single-draw" generalization bound due to [4].

For a $(\sigma, c)$-sub-gamma under $\mu$, we can take $\psi(\beta)=\frac{\beta^{2} \sigma^{2}}{2(1-\beta c)}$ for every $\beta$ such that $\beta \in\left(0, \frac{1}{c}\right)$ and $\psi^{*-1}(y)=\sqrt{2 \sigma^{2} y}+c y[33, \S 2.4]$, which gives the following result:

Corollary 8. If $\ell(w, Z)$ is $(\sigma, c)$-sub-gamma under $\mu$ for all $w \in \mathcal{W}$, then

$$
\mathbb{E}_{S W}[\mathrm{~g}(W, S)] \leq \sqrt{2 \sigma^{2} I(S ; W) / n}+c I(S ; W) / n .
$$

Fixing $\beta=1$ in (6), we recover [27, Corollary 5]:

Corollary 9. Consider the setting in Theorem 5. If the loss $\ell$ is $(\sigma, c)$-sub-gamma with $c<1$, then with probability of at least $1-\delta$ over the choice of $S \sim \mu^{\otimes n}$, for all distributions $P \ll Q$ over $\mathcal{W}$,

$$
\mathbb{E}_{P}[\mathrm{~g}(W, S)] \leq \frac{1}{n}\left(D(P \| Q)+\ln \frac{1}{\delta}\right)+\frac{\sigma^{2}}{2(1-c)} .
$$

The condition $c<1$ guarantees that $\beta=1 \in\left(0, \frac{1}{c}\right)$ when the sub-gamma condition in Proposition 1 item 8 ) is satisfied. In the limit $c \rightarrow 0_{+}$, a sub-gamma loss reduces to the sub-Gaussian loss $[33, \S 2.4]$, and we recover [27, Corollary 4].

For the sub-Gaussian loss, fixing $\beta=1 / \sqrt{n}$ in (6), the second term $\psi(\beta) / \beta$ decays with increasing $n$, but then the first term will have a slower decay of $1 / \sqrt{n}$ instead of $1 / n$.

We can also optimize $\beta$ in (6) at a small cost using the union bound:

Proposition 10. Consider the setting in Theorem 5. If $\ell(w, Z)$ is $\sigma$-sub-Gaussian under $\mu$ for all $w \in \mathcal{W}$, then for any constants $\alpha>1$ and $v>0$, and any $\delta \in(0,1]$, for all $\beta \in(0, v]$, with probability of at least $1-\delta$, we have

$$
\mathbb{E}_{P}[\mathrm{~g}(W, S)] \leq \frac{\alpha}{n \beta}\left(D(P \| Q)+\ln \frac{\log _{\alpha} \sqrt{n}+K}{\delta}\right)+\frac{\beta \sigma^{2}}{2}
$$

where $K=\max \left\{\log _{\alpha}\left(\frac{v \sigma}{\sqrt{2 \alpha}}\right), 0\right\}+e$.

\subsection{The conditional mutual information (CMI) bound}

One drawback of the mutual information-based bounds in Corollaries 7 and 8 is that $I(S ; W)$ can be unbounded in many practical situations of interest [5,7]. CMI-based bounds [7,8] address this issue by conditioning on a superset of the training sample called the supersample, in effect, normalizing the information content of each datum to one bit. As nicely articulated by Steinke and Zakynthinou [7], intuitively, the difference between the CMI- and MI- based approaches is that 
between "recognizing" vs. "reconstructing" the input, given the output of the algorithm. Recognizing the input is formalized by considering a i.i.d. supersample $\tilde{Z} \in \mathcal{Z}^{n \times 2}$ consisting of $n \times 2$ data points, which comprises of $n$ "true" input data points mixed with $n$ "ghost" data points. A selector variable $U \in\{0,1\}^{n}$ chooses the input samples from the supersample, uniformly at random. Given the output of the algorithm, CMI then measures how well it is possible to distinguish the true inputs from their ghosts. We note the following definition:

Definition 11 (CMI of an algorithm $P_{W \mid S}$ [7]). Let $\mu$ be a probability distribution on $\mathcal{Z}$ and let $\tilde{Z} \in \mathcal{Z}^{n \times 2}$ consist of $2 n$ i.i.d. samples drawn from $\mu$. Let $U=\left(U_{1}, \ldots, U_{n}\right) \in\{0,1\}^{n}$ be uniformly random and independent from $\tilde{Z}$ and the randomness of the algorithm. Define $S:=\tilde{Z}_{U} \in \mathcal{Z}^{n}$ by $\left(\tilde{Z}_{U}\right)_{i}=\tilde{Z}_{i, U_{i}+1}$ for all $i \in[n]$, i.e., $S$ is the subset of $\tilde{Z}$ indexed by $U$. Then the conditional mutual information (CMI) of an algorithm $P_{W \mid S}$ w.r.t. $\mu$ is

$$
\mathrm{CMI}_{\mu}\left(P_{W \mid S}\right):=I(W ; U \mid \tilde{Z}) \text {. }
$$

Since $S$ is a deterministic function of $\tilde{Z} U$, we have $W-\tilde{Z} U-S$. Also $W-S-\tilde{Z} U$ since $W$ depends on $\tilde{Z} U$ only through $S$. Together, this implies

$$
I(S ; W)=I(\tilde{Z} U ; W)=I(W ; \tilde{Z})+I(W ; U \mid \tilde{Z}) .
$$

Suppose that we observe the output $W$ and wish to identify $S$ given access to $\tilde{Z}$. For any estimator $\widehat{U}=\xi(W, \tilde{Z})$ of $U$, by Fano's inequality we have

$$
\inf _{\xi} \operatorname{Pr}(\xi(W, \tilde{Z}) \neq U) \geq 1-\frac{I(W ; U \mid \tilde{Z})+\log 2}{n \log 2} .
$$

$I(W ; U \mid \tilde{Z})$ thus upper-bounds the probability of successfully identifying $U$ from $\widehat{U}$.

In $[7$, Theorem $2(1)]$, it is shown that for a $[0,1]$-valued loss,

$$
\mathbb{E}_{S W}[\mathrm{~g}(W, S)] \leq \sqrt{2 \cdot \mathrm{CMI}_{\mu}\left(P_{W \mid S}\right) / n} .
$$

Unlike the mutual information $I(S ; W)$ that can be potentially unbounded, $\mathrm{CMI}_{\mu}\left(P_{W \mid S}\right)$ is bounded above by $n \log 2$.

We give a PAC-Bayesian version of the CMI bound in Proposition 12 . Let $\bar{U}=\left(\bar{U}_{1}, \ldots, \bar{U}_{n}\right)$ be a vector obtained by inverting all the bits of $U$, and define $\bar{S}=\tilde{Z}_{\bar{U}} . S$ and $\bar{S}$ have a common marginal distribution, $\mu^{\otimes n}$. The algorithm maps the input $S=\tilde{Z}_{U}$ to a random element $W$ of $\mathcal{W}$. Since $\bar{S} \Perp W$, we can define the generalization error as $\mathrm{g}(W, \tilde{Z}, U):=L_{\bar{S}}(W)-L_{S}(W)$, where $L_{\bar{S}}(w):=\frac{1}{n} \sum_{i=1}^{n} \ell\left(w,\left(\tilde{Z}_{\bar{U}}\right)_{i}\right)$, and $L_{S}(w):=\frac{1}{n} \sum_{i=1}^{n} \ell\left(w,\left(\tilde{Z}_{U}\right)_{i}\right)$. Given a realization of the supersample $\tilde{Z}=\tilde{z}$ and selector variable $U=u$, we write $Q \equiv Q_{W \mid \tilde{z}}$ and $P \equiv P_{W \mid \tilde{z}, u}$ for, resp., the prior and the posterior distribution. Then the following bounds hold for all such prior and posterior distributions:

Proposition 12. For any [0,1]-valued loss function $\ell$, for any $\beta>0$ and $\delta \in(0,1]$, with probability of at least $1-\delta$ over a draw of $\tilde{Z}, U$ as defined above, we have:

$$
\mathbb{E}_{P}[\mathrm{~g}(W, \tilde{Z}, U)] \leq \frac{1}{n \beta}\left(D(P \| Q)+\ln \frac{1}{\delta}\right)+\frac{\beta}{2} .
$$


Moreover, we have the following bound in expectation:

$$
\mathbb{E}_{W, \tilde{Z}, U}[\mathrm{~g}(W, \tilde{Z}, U)] \leq \sqrt{\frac{2 \cdot D\left(P \| Q \mid P_{\tilde{Z}, U}\right)}{n}} .
$$

Using the same reasoning as earlier, supplanting the associated oracle prior recovers the bound in expectation $\sqrt{2 \cdot \mathrm{CMI}_{\mu}\left(P_{W \mid S}\right) / n}$ in [7, Theorem 2(1)].

\subsection{Recovering classical PAC-Bayesian bounds}

By Proposition 1 item 6), for a $\{0,1\}$-valued loss, we have

$$
M_{\beta}(w)=-\beta^{-1} \ln \left(1-\left(1-e^{-\beta}\right) L_{\mu}(w)\right)=: \Phi_{\beta}\left(L_{\mu}(w)\right) .
$$

$\Phi_{\beta}$ is an increasing one-to-one mapping of the unit interval onto itself, and is convex for $\beta>0$. The inverse of $\Phi_{\beta}$ is given by $\Phi_{\beta}^{-1}(x)=\frac{1-e^{-\beta x}}{1-e^{-\beta}}$, and we recover Catoni's PAC-Bayesian bound:

Corollary 13 (Catoni's bound [21, Theorem 1.2.6]). For any $\{0,1\}$-valued loss $\ell$, any distribution $\mu$, prior $Q \in \mathcal{M}(\mathcal{W})$, any real $\beta>0$, and any $\delta \in(0,1]$, with probability of at least $1-\delta$ over $S \sim \mu^{\otimes n}$, we have for all $P \ll Q$ over $\mathcal{W}$ :

$$
\mathbb{E}_{P}\left[L_{\mu}(W)\right] \leq \Phi_{\beta}^{-1}\left\{\mathbb{E}_{P}\left[L_{S}(W)\right]+\frac{1}{n \beta}\left(D(P \| Q)+\ln \frac{1}{\delta}\right)\right\}
$$

Using $1 \leq \beta\left(1-e^{-\beta}\right)^{-1} \leq\left(1-\frac{\beta}{2}\right)^{-1}$, we have

$$
\begin{aligned}
\mathbb{E}_{P}\left[L_{\mu}(W)\right] & \leq \Phi_{\beta}^{-1}\left\{\mathbb{E}_{P}\left[L_{S}(W)\right]+\frac{1}{n \beta}\left(D(P \| Q)+\ln \frac{1}{\delta}\right)\right\} \\
& \leq \frac{\beta}{1-e^{-\beta}}\left[\mathbb{E}_{P}\left[L_{S}(W)\right]+\frac{1}{n \beta}\left(D(P \| Q)+\ln \frac{1}{\delta}\right)\right] \\
& \leq \frac{1}{1-\frac{\beta}{2}}\left[\mathbb{E}_{P}\left[L_{S}(W)\right]+\frac{1}{n \beta}\left(D(P \| Q)+\ln \frac{1}{\delta}\right)\right]
\end{aligned}
$$

(10) and (11) recover, resp., Catoni's [21, Theorem 1.2.1] and McAllester's "Linear PAC-Bayes bound" [18, Theorem 2], where for the latter we additionally require that $\beta<2$.

For loss functions bounded in $[0,1]$, we elaborate in Appendix B.3 on other approximations that lead to several well-known PAC-Bayesian inequalities such as the "PAC-Bayes-KL inequality" $[22,35]$.

Remark 14 (Related work). A variation of the IEI for the special case of the 0-1 loss appears in the monograph by Catoni [21, Eq.1.2], and has been rediscovered more recently for the sub-Gaussian loss in [36, 37]. The statements of [36, Corollary 3, Eq. 20] and [37, Corollary 6, Eq. 95] which are analogues of our Proposition 10 and Proposition 12, Eq. 8, resp., are incorrect as they assume that $\beta$ can be optimized "for free," when in fact we have to pay a union bound price for optimizing $\beta$, which is selected before the draw of the training sample. We also note two related works that focus exclusively on unifying either PAC-Bayesian bounds for the 0-1 loss [38], or information-theoretic bounds for the sub-Gaussian loss [39]. 


\section{Differentially private data-dependent priors}

A PAC-Bayesian bound such as (3) stipulates that the prior $Q$ be chosen before the draw of the training sample $S$. $Q$ may depend on the data generating distribution $\mu$ [40]. However, our access to $\mu$ is only through $S$. To have a good control over the KL term in (3), it is desirable that $Q$ be "aligned" with the data-dependent posterior $P$. One way to achieve this goal is to choose $Q$ based on $S$ in a differentially private fashion so that $Q$ is stable to local perturbations in $S$ [41]. We can then treat $Q$ "as if" it is independent of $S$. Here, the key quantity of interest is the approximate max-information between the input $S$ and the data-dependent prior. We shall make these notions precise.

For $\alpha \geq 0$, the $\alpha$-approximate max-divergence is defined as

$$
D_{\infty}^{\alpha}(P \| Q)=\ln \sup _{\mathcal{O} \subseteq \mathcal{X}: P(\mathcal{O})>\alpha} \frac{P(\mathcal{O})-\alpha}{Q(\mathcal{O})}
$$

The max-divergence $D_{\infty}(P \| Q)$ is defined as $D_{\infty}^{\alpha}(P \| Q)$ for $\alpha=0$. For a pair of variables $(X, Y)$ with joint law $P_{X Y}$ and marginals $P_{X}$ and $P_{Y}$, the $\alpha$-approximate max-information between $X$ and $Y$ is defined as $I_{\infty}^{\alpha}(X ; Y)=D_{\infty}^{\alpha}\left(P_{X Y} \| P_{X} \otimes P_{Y}\right)$. The max-information $I_{\infty}(X ; Y)$ is defined to be $I_{\infty}^{\alpha}(X ; Y)$ for $\alpha=0$. $I_{\infty}(X ; Y)$ is an upper bound on the ordinary mutual information $I(X ; Y)$ [13].

Definition 15 (Differential Privacy [42]). For any $\epsilon>0$ and $\delta \in[0,1]$, an algorithm $P_{W \mid S}$ is said to be $(\epsilon, \delta)$-differentially private if for all pairs of datasets $s, s^{\prime} \in \mathcal{Z}^{n}$ that differ in a single element, $D_{\infty}^{\delta}\left(P_{W \mid S=s} \| P_{W \mid S=s^{\prime}}\right) \leq \epsilon$. The case $\delta=0$ is called pure differential privacy.

Definition 16 (Max-Information of an algorithm [13]). We say that an algorithm $P_{W \mid S}$ has $\alpha$ approximate max-information of $k$, denoted as $I_{\infty, \mu}^{\alpha}\left(P_{W \mid S}, n\right) \leq k$, if for every distribution $\mu$ over $\mathcal{Z}$, we have $I_{\infty}^{\alpha}(S ; W) \leq k$ when $S \sim \mu^{\otimes n}$.

It follows from the definition of $\alpha$-approximate max-information that if an algorithm $P_{W \mid S}$ has bounded approximate max-information, then we can control the probability of "bad events" that may arise as a result of the dependence of the output $W$ on the input $S$ [13]. Let $S^{\prime} \Perp W$ be an independent sample with the same distribution as $S$. If for some $\alpha \geq 0, I_{\infty}^{\alpha}(S ; W)=k$, then for any event $\mathcal{O} \subseteq \mathcal{Z}^{n} \times \mathcal{W}$, we have

$$
\operatorname{Pr}((S, W) \in \mathcal{O}) \leq e^{k} \cdot \operatorname{Pr}\left(\left(S^{\prime}, W\right) \in \mathcal{O}\right)+\alpha .
$$

Pure differential privacy implies a bound on the approximate max-information:

Theorem 17 (Pure differential privacy and $\alpha$-approximate max-information [13, Theorem 20]). If $P_{W \mid S}$ is an $(\epsilon, 0)$-differentially private algorithm, then $I_{\infty, \mu}\left(P_{W \mid S}, n\right) \leq n \epsilon$, and for any $\alpha>0$, $I_{\infty, \mu}^{\alpha}\left(P_{W \mid S}, n\right) \leq n \epsilon^{2} / 2+\epsilon \sqrt{n \ln (2 / \alpha) / 2}$.

Remark 18. The result above is extended to $(\epsilon, \delta)$-differential privacy in [14, Theorem 3.1]: If $P_{W \mid S}$ is an $(\epsilon, \delta)$-differentially private algorithm for $\epsilon \in(0,1 / 2]$ and $\delta \in(0, \epsilon)$, then for $\alpha=O(n \sqrt{\delta / \epsilon})$, $I_{\infty, \mu}^{\alpha}(\mathcal{A}, n)=O\left(n \epsilon^{2}+n \sqrt{\delta / \epsilon}\right)$. 
Proposition 19. Consider the setting in Theorem 5. Let $Q^{0} \in \mathcal{K}(\mathcal{S}, \mathcal{W})$ be an $(\epsilon, 0)$-differentially private algorithm. Then with probability of at least $1-\delta$ over the choice of $S \sim \mu^{\otimes n}$, for all $P \in \mathcal{M}(\mathcal{W})$

$$
\mathbb{E}_{P}\left[M_{\beta}(W)\right] \leq \mathbb{E}_{P}\left[L_{S}(W)\right]+\frac{D\left(P \| Q^{0}(S)\right)+f_{n}(\delta, \epsilon)}{n \beta} .
$$

where $f_{n}(\delta, \epsilon):=\ln \frac{2}{\delta}+\frac{n \epsilon^{2}}{2}+\epsilon \sqrt{\frac{n}{2} \ln \frac{4}{\delta}}$.

By Remark 18, the result above can be extended to $(\epsilon, \delta)$-differentially private priors. Proposition 19 is similar in spirit to the traditional PAC-Bayesian bounds in [41, Theorem 4.2], and [29, Eq. 7], which, however, either apply only when the loss is bounded in $[0,1]$, or entails approximating a suitable exponential moment involving the true risk. We can also bound the expected generalization error. The next result follows from Theorems 17 and 6:

Corollary 20. Consider the setting in Theorem 6. Let $Q^{0} \in \mathcal{K}(\mathcal{S}, \mathcal{W})$ be an $(\epsilon, 0)$-differentially private algorithm. Then with probability of at least $1-\delta$ over a draw of the sample $S$, for all $P$, we have

$$
\mathbb{E}_{P}[\mathrm{~g}(W, S)] \leq \frac{D\left(P \| Q^{0}(S)\right)+f_{n}(\delta, \epsilon)}{n \beta}+\frac{\psi(\beta)}{\beta}
$$

where $f_{n}(\delta, \epsilon):=\ln \frac{2}{\delta}+\frac{n \epsilon^{2}}{2}+\epsilon \sqrt{\frac{n}{2} \ln \frac{4}{\delta}}$.

The main advantage of the max-information formulation is that we can get high probability guarantees at the cost of a $O\left(n \epsilon^{2}+\epsilon \sqrt{n \ln 1 / \delta}\right)$ correction term. This cost is compensated for by a lower KL complexity since the prior is more "aligned" with the data-dependent posterior than when chosen independently of the data. As is well-known [13,15], a small mutual information between the data and the prior will not ensure that bad events will happen with low probability.

\section{Information complexity minimization}

Given any prior $Q$, minimizing the right hand side of (3) gives rise to the Information Complexity Minimization (ICM) framework [20,34]. Concretely, for a given prior $Q$ and hypothesis set $\mathcal{G} \subseteq$ $\mathcal{M}(\mathcal{W})$, define the Optimal Information Complexity (OIC) at a given $\beta$ as

$$
\mathrm{OIC}_{\mathcal{G}}^{\beta}:=\inf _{P \in \mathcal{G}}\left\{\mathbb{E}_{P}\left[L_{S}(W)\right]+(n \beta)^{-1} D(P \| Q)\right\} .
$$

When $\mathcal{G}=\mathcal{M}(\mathcal{W})$, applying Lemma 3 to $f(w)=n L_{S}(w)$, and writing $\beta$ for $n \beta$, we obtain the Gibbs measure, $P^{\star}$, in which case the OIC evaluates to the (extended) stochastic complexity $[43,44]$

$$
-\beta^{-1} \ln \mathbb{E}_{Q}\left[e^{-\beta L_{S}(W)}\right] .
$$

The latter in turn coincides with the negative log-marginal likelihood for $\beta=1$ and the logarithmic loss function $[20,34,45]$.

We briefly discuss two practical examples of ICM for learning with neural networks (NNs), namely, PAC-Bayes-SGD [31] and Entropy-SGD [46], which can be viewed as optimization schemes that search for a "flat" minimum of the empirical loss surface [32]. We also show a PAC-Bayesian bound motivated by an Occam factor argument [47] in relation to flat minima. 


\subsection{PAC-Bayes-SGD}

$P A C$-Bayes-SGD is an approach to computing generalization bounds for overparameterized NN classifiers trained with stochastic gradient descent (SGD) [31]. These bounds are obtained by retraining the network using an objective derived from a PAC-Bayes bound, starting from the solution found by SGD (or in fact any other procedure) for the training loss $L_{S}(w)$ w.r.t. $w$. The underlying hypothesis is that SGD finds "good" solutions that generalize well on unseen data only if such solutions are surrounded by a large volume of equally good solutions. The method draws on an earlier work by Langford and Caruana [48], and is closely related to the bits-back argument due to Hinton and van Camp [49] (see Appendix A). We show how Catoni's bound in Corollary 13 can be used to derive a PAC-Bayes-SGD objective.

Consider a binary classification setting with examples domain $\mathcal{Z}=\mathcal{X} \times\{0,1\}$ and loss $\ell: \mathbb{R}^{k} \times$ $\mathcal{Z} \rightarrow\{0,1\}$. Each $w \in \mathcal{W}$ corresponds to a classifier $f_{w}: \mathcal{X} \rightarrow\{0,1\}$ that can be interpreted as a deterministic NN with parameters in $\mathbb{R}^{k}$. For trainable parameters $w_{P} \in \mathbb{R}^{k}, \gamma \in \mathbb{R}_{+}^{k}, \lambda \in \mathbb{R}_{+}$, let $\mathcal{G}$ be the set of all Gaussian posteriors of the form $P=\mathcal{N}\left(w_{P}, \operatorname{diag}(\gamma)\right)$ and let $Q=\mathcal{N}\left(w_{0}, \lambda I_{k}\right)$ be a prior centered at a non-trainable random initialization, $w_{0} \in \mathbb{R}^{k}$. We can use a convex surrogate of the 0-1 loss, and the reparameterization trick $w=w_{P}+\nu \odot \sqrt{\gamma}, \nu \sim \mathcal{N}\left(0, I_{k}\right)$ [50] to compute an unbiased estimate of the gradient of the PAC-Bayes bound in Corollary 13 w.r.t. the parameters $w_{P}, \gamma, \lambda$ and $\beta$. Computing the expectation $\mathbb{E}_{P}\left[L_{S}\left(f_{W}\right)\right]$ is difficult in practice. Instead, we can use

a Monte Carlo estimate $\hat{L}_{S}\left(f_{W}\right)=\frac{1}{m} \sum_{i=1}^{m} L_{S}\left(f_{W_{i}}\right)$, where $W_{i} \stackrel{\text { i.i.d. }}{\sim} P$. Then Corollary 13 takes the form: For any $\delta, \delta^{\prime} \in(0,1)$, fixed $\alpha>1, c \in(0,1), b \in \mathbb{N}$, and $m, n \in \mathbb{N}$, with probability of at least $1-\delta-\delta^{\prime}$ over a draw of $S \sim \mu^{\otimes n}$ and $W \sim(P)^{\otimes m}$,

$$
\mathbb{E}_{P}\left[L_{\mu}\left(f_{W}\right)\right] \leq \inf _{P \in \mathcal{G}, \beta>1, \lambda \in(0, c)} \Phi_{\beta}^{-1}\left\{\hat{L}_{S}\left(f_{W}\right)+\frac{\alpha}{n \beta} D(P \| Q)+R\left(\lambda, \beta ; \delta, \delta^{\prime}\right)\right\},
$$

where $R=\frac{2 \alpha}{n \beta} \ln \left[\frac{\ln \alpha^{2} \beta n}{\ln \alpha}\right]+\frac{\alpha}{n \beta} \ln \left[\frac{\pi^{2} b^{2}}{6 \delta}\left(\ln \frac{c}{\lambda}\right)^{2}\right]+\sqrt{\frac{1}{2 m} \ln \frac{2}{\delta^{\prime}}}$ accounts for the cost of optimizing the parameters $\beta, \lambda$, and using the Monte Carlo estimate of the empirical risk. For large $n, m, R$ is negligible, and the optimization is dominated by the IC term, $\hat{L}_{S}\left(f_{W}\right)+\alpha(n \beta)^{-1} D(P \| Q)$.

\subsection{Entropy-SGD}

A related approach is Entropy-SGD [46], which directly minimizes the stochastic complexity,

$$
-\beta^{-1} \ln \mathbb{E}_{Q} e^{-\beta L_{S}(W)} .
$$

This, however, entails optimizing the prior $Q$, when ideally $Q$ must be chosen before the draw of the training sample $S$. We can sample $Q$ instead in a differentially private fashion, and this forms the basis of the Entropy-SGLD algorithm [51]. For $Q=\mathcal{N}\left(w,(\beta \gamma)^{-1} I_{k}\right)$, the stochastic complexity can be equivalently written (up to constant terms) as

$$
-\beta^{-1} \ln \int_{w^{\prime} \in \mathbb{R}^{k}} e^{-\beta\left[L_{S}\left(w^{\prime}\right)+\frac{\gamma}{2}\left\|w-w^{\prime}\right\|^{2}\right]} \mathrm{d} w^{\prime},
$$

which can be interpreted as a measure of flatness of the loss surface that measures the log-volume of low-loss parameter configurations around $w$. From the perspective of ICM, both Entropy- and PAC-Bayes- SGD can be viewed as optimization schemes that search for flat minima solutions. 


\subsection{PAC-Bayes and Occam factor}

Lemma 21 gives the form of the optimal posterior under a quadratic approximation of the loss around a local minimizer:

Lemma 21. Consider a quadratic approximation of the training loss around a local minimizer $w_{P}$, $\tilde{L}_{S}(w)=\frac{1}{2}\left(w-w_{P}\right)^{\top} H\left(w-w_{P}\right)$ where $H=\left.\nabla^{2} L_{S}(w)\right|_{w=w_{P}}$, a fixed prior $Q=\mathcal{N}\left(w_{Q}, \lambda^{-1} I_{k}\right)$, and a posterior distribution of the form $P=\mathcal{N}\left(w_{P}, \Sigma_{P}\right)$. Then the solution to the convex optimization problem $\min _{\Sigma_{P}} \mathbb{E}_{P}\left[\tilde{L}_{S}(W)\right]+(n \beta)^{-1} D(P \| Q)$, is given by $\Sigma_{P}^{\star}=H_{\lambda}^{-1}$, where $H_{\lambda}:=\left(n \beta H+\lambda I_{k}\right)$. Here we assume $\lambda>0$ is sufficiently large so that $H_{\lambda}$ is positive definite.

We can use a posterior of the form $P=\mathcal{N}\left(w_{P}, H_{\lambda}^{-1}\right)$ to get the following PAC-Bayesian bound that incorporates second-order curvature information of the training loss:

Proposition 22. Let $\left\{\lambda_{i}\right\}_{i=1}^{k}$ be the eigenvalues of $H_{\lambda}$ and suppose that $\lambda_{i} \geq \lambda>0$ for all $i$. Let $Q=\mathcal{N}\left(w_{Q}, \lambda^{-1} I_{k}\right)$ be a prior, and let $P=\mathcal{N}\left(w_{P}, H_{\lambda}^{-1}\right)$. Then with probability of at least $1-\delta$ over a draw of the sample $S$, we have

$$
\mathbb{E}_{P}\left[M_{\beta}(W)\right] \leq \mathbb{E}_{P}\left[L_{S}(W)\right]+\frac{1}{n \beta} \ln \frac{1}{\delta}+\frac{1}{n \beta}\left(\frac{\lambda}{2}\left\|w_{Q}-w_{P}\right\|^{2}+\frac{1}{2} \sum_{i=1}^{k} \ln \frac{\lambda_{i}}{\lambda}\right) .
$$

Notably, the log-ratio term in (14),

$$
\frac{1}{2} \sum_{i=1}^{k} \ln \frac{\lambda_{i}}{\lambda}=-\ln \sqrt{\operatorname{det} \frac{\lambda}{H_{\lambda}}}
$$

is the negative logarithm of the Occam factor $[47,52,53]$. The Occam factor can be interpreted as the fraction of the prior parameter space that is consistent with the training data. The log-Occam factor is the differential entropy associated with a Gaussian posterior with scaled covariance $\lambda\left(H_{\lambda}\right)^{-1}$, and can be interpreted as the amount of information we gain about the model's parameters after seeing the training data. From the perspective of ICM, minimizing the right hand side of (14) w.r.t. the posterior leads to solutions with higher entropy and hence wider minima.

\section{Discussion}

We presented a unified treatment of PAC-Bayesian and information-theoretic generalization bounds starting from a fundamental information-theoretic inequality. Besides recovering several well-known bounds in the literature, we also obtained new bounds for data-dependent priors and unbounded loss functions. The bounds we studied are along the notion that bounded information (between the training data and the output hypothesis) implies learning. On the other hand, it is known that learning does not imply bounded information $[4,54]$. In particular, the information revealed by a learning algorithm about its input can be unbounded even for hypothesis classes of $\mathrm{VC}$ dimension 1. A result in a similar vein appears in the PAC-Bayesian framework [55]. Identifying the common structural properties of these negative results in the information-theoretic and PAC-Bayesian frameworks is an important avenue for further investigation. 


\section{Acknowledgment}

This project has received funding from the European Research Council (ERC) under the EU's Horizon 2020 research and innovation programme (grant agreement $\mathrm{n}^{\mathrm{o}} 757983$ ).

\section{References}

[1] D. Russo and J. Zou, "Controlling bias in adaptive data analysis using information theory," in Proceedings of the 19th International Conference on Artificial Intelligence and Statistics (AISTATS), 2016, pp. 1232-1240.

[2] A. Xu and M. Raginsky, "Information-theoretic analysis of generalization capability of learning algorithms," in Advances in Neural Information Processing Systems, 2017, pp. 2524-2533.

[3] J. Jiao, Y. Han, and T. Weissman, "Dependence measures bounding the exploration bias for general measurements," in Proceedings of the IEEE International Symposium on Information Theory (ISIT). IEEE, 2017, pp. 1475-1479.

[4] R. Bassily, S. Moran, I. Nachum, J. Shafer, and A. Yehudayoff, "Learners that use little information," in International Conference on Algorithmic Learning Theory (ALT), 2018, pp. 25-55.

[5] Y. Bu, S. Zou, and V. V. Veeravalli, "Tightening mutual information based bounds on generalization error," in Proceedings of the IEEE International Symposium on Information Theory (ISIT). IEEE, 2019, pp. 587-591.

[6] I. Issa, A. R. Esposito, and M. Gastpar, "Strengthened information-theoretic bounds on the generalization error," in Proceedings of the IEEE International Symposium on Information Theory (ISIT). IEEE, 2019, pp. 582-586.

[7] T. Steinke and L. Zakynthinou, "Reasoning about generalization via conditional mutual information," in Conference On Learning Theory, 2020, pp. 3437-3452.

[8] — , "Open problem: Information complexity of VC learning," in Conference on Learning Theory, 2020, pp. 3857-3863.

[9] M. Haghifam, J. Negrea, A. Khisti, D. M. Roy, and G. K. Dziugaite, "Sharpened generalization bounds based on conditional mutual information and an application to noisy, iterative algorithms," Advances in Neural Information Processing Systems, vol. 33, pp. 9925-9935, 2020.

[10] O. Bousquet and A. Elisseeff, "Stability and generalization," Journal of Machine Learning Research, vol. 2, no. Mar, pp. 499-526, 2002.

[11] S. Shalev-Shwartz, O. Shamir, N. Srebro, and K. Sridharan, "Learnability, stability and uniform convergence," The Journal of Machine Learning Research, vol. 11, pp. 2635-2670, 2010.

[12] R. Bassily, K. Nissim, A. Smith, T. Steinke, U. Stemmer, and J. Ullman, "Algorithmic stability for adaptive data analysis," in Proceedings of the 48th Annual ACM Symposium on Theory of Computing (STOC), 2016, pp. 1046-1059. 
[13] C. Dwork, V. Feldman, M. Hardt, T. Pitassi, O. Reingold, and A. Roth, "Generalization in adaptive data analysis and holdout reuse," in Advances in Neural Information Processing Systems, 2015, pp. 2350-2358.

[14] R. Rogers, A. Roth, A. Smith, and O. Thakkar, "Max-information, differential privacy, and post-selection hypothesis testing," in 57th Annual Symposium on Foundations of Computer Science (FOCS). IEEE, 2016, pp. 487-494.

[15] V. Feldman and T. Steinke, "Calibrating noise to variance in adaptive data analysis," in Conference On Learning Theory, 2018, pp. 535-544.

[16] D. A. McAllester, "PAC-Bayesian model averaging," in Proceedings of the 12th Annual Conference on Computational Learning Theory. ACM, 1999, pp. 164-170.

[17] —_, "Some PAC-Bayesian theorems," Machine Learning, vol. 37, no. 3, pp. 355-363, 1999.

[18] — _ "A PAC-Bayesian tutorial with a dropout bound," arXiv preprint arXiv:130\%.2118, 2013.

[19] S. Shalev-Shwartz and S. Ben-David, Understanding machine learning: From theory to algorithms. Cambridge University Press, 2014.

[20] T. Zhang, "Information-theoretic upper and lower bounds for statistical estimation," IEEE Transactions on Information Theory, vol. 52, no. 4, pp. 1307-1321, 2006.

[21] O. Catoni, PAC-Bayesian Supervised Classification: The Thermodynamics of Statistical Learning. Institute of Mathematical Statistics, 2007, vol. 56.

[22] A. Maurer, "A note on the PAC Bayesian theorem," arXiv preprint cs/0411099, 2004.

[23] T. van Erven, "PAC-Bayes mini-tutorial: A continuous union bound," arXiv preprint arXiv:1405.1580, 2014.

[24] P. D. Grünwald and N. A. Mehta, "Fast rates for general unbounded loss functions: From ERM to generalized Bayes." Journal of Machine Learning Research, vol. 21, no. 56, pp. 1-80, 2020.

[25] P. Alquier, J. Ridgway, and N. Chopin, "On the properties of variational approximations of Gibbs posteriors," The Journal of Machine Learning Research, vol. 17, no. 1, pp. 8374-8414, 2016.

[26] P. Germain, A. Lacasse, F. Laviolette, and M. Marchand, "PAC-Bayesian learning of linear classifiers," in Proceedings of the 26th International Conference on Machine Learning (ICML), 2009, pp. 353-360.

[27] P. Germain, F. Bach, A. Lacoste, and S. Lacoste-Julien, "PAC-Bayesian theory meets Bayesian inference," in Advances in Neural Information Processing Systems, 2016, pp. 1884-1892.

[28] N. Thiemann, C. Igel, O. Wintenberger, and Y. Seldin, "A strongly quasiconvex PAC-Bayesian bound," in International Conference on Algorithmic Learning Theory (ALT), 2017, pp. 466492.

[29] O. Rivasplata, I. Kuzborskij, C. Szepesvári, and J. Shawe-Taylor, "PAC-Bayes analysis beyond the usual bounds," in Advances in Neural Information Processing Systems, vol. 33, 2020. 
[30] I. Kuzborskij, N. Cesa-Bianchi, and C. Szepesvári, "Distribution-dependent analysis of GibbsERM principle," in Conference on Learning Theory, 2019, pp. 2028-2054.

[31] G. K. Dziugaite and D. M. Roy, "Computing nonvacuous generalization bounds for deep (stochastic) neural networks with many more parameters than training data," in Proceedings of the 33rd Conference on Uncertainty in Artificial Intelligence (UAI), 2017.

[32] S. Hochreiter and J. Schmidhuber, "Flat minima," Neural Computation, vol. 9, no. 1, pp. 1-42, 1997.

[33] S. Boucheron, G. Lugosi, and P. Massart, Concentration inequalities: A nonasymptotic theory of independence. Oxford University Press, 2013.

[34] T. Zhang, "From $\varepsilon$-entropy to KL-entropy: Analysis of minimum information complexity density estimation," The Annals of Statistics, vol. 34, no. 5, pp. 2180-2210, 2006.

[35] M. Seeger, "PAC-Bayesian generalisation error bounds for Gaussian process classification," Journal of Machine Learning Research, vol. 3, no. Oct, pp. 233-269, 2002.

[36] F. Hellström and G. Durisi, "Generalization error bounds via $m$ th central moments of the information density," in Proceedings of the IEEE International Symposium on Information Theory (ISIT). IEEE, 2020, pp. 2741-2746.

[37] - "Generalization bounds via information density and conditional information density," IEEE Journal on Selected Areas in Information Theory, pp. 824-839, 2020.

[38] A. Blum and J. Langford, "PAC-MDL bounds," in Learning theory and kernel machines. Springer, 2003, pp. 344-357.

[39] H. Hafez-Kolahi, Z. Golgooni, S. Kasaei, and M. Soleymani, "Conditioning and processing: Techniques to improve information-theoretic generalization bounds," in Advances in Neural Information Processing Systems, vol. 33, 2020.

[40] G. Lever, F. Laviolette, and J. Shawe-Taylor, "Tighter PAC-Bayes bounds through distributiondependent priors," Theoretical Computer Science, vol. 473, pp. 4-28, 2013.

[41] G. K. Dziugaite and D. M. Roy, "Data-dependent PAC-Bayes priors via differential privacy," in Advances in Neural Information Processing Systems, 2018, pp. 8430-8441.

[42] C. Dwork and A. Roth, "The algorithmic foundations of differential privacy," Foundations and Trends@ in Theoretical Computer Science, vol. 9, no. 3-4, pp. 211-407, 2014.

[43] J. Rissanen, Stochastic complexity in statistical inquiry. World scientific, 1989.

[44] K. Yamanishi, "A decision-theoretic extension of stochastic complexity and its applications to learning," IEEE Transactions on Information Theory, vol. 44, no. 4, pp. 1424-1439, 1998.

[45] A. R. Barron and T. M. Cover, "Minimum complexity density estimation," IEEE Transactions on Information Theory, vol. 37, no. 4, pp. 1034-1054, 1991. 
[46] P. Chaudhari, A. Choromanska, S. Soatto, Y. LeCun, C. Baldassi, C. Borgs, J. Chayes, L. Sagun, and R. Zecchina, "Entropy-SGD: Biasing gradient descent into wide valleys," in International Conference on Learning Representations, 2017.

[47] D. J. C. MacKay, "A practical Bayesian framework for backpropagation networks," Neural computation, vol. 4, no. 3, pp. 448-472, 1992.

[48] J. Langford and R. Caruana, "(Not) bounding the true error," in Advances in Neural Information Processing Systems, 2002, pp. 809-816.

[49] G. E. Hinton and D. van Camp, "Keeping neural networks simple by minimising the description length of weights," in Conference On Learning Theory, 1993, pp. 5-13.

[50] C. Blundell, J. Cornebise, K. Kavukcuoglu, and D. Wierstra, "Weight uncertainty in neural networks," in Proceedings of the 32nd International Conference on Machine Learning (ICML), 2015, pp. 1613-1622.

[51] G. K. Dziugaite and D. M. Roy, "Entropy-SGD optimizes the prior of a PAC-Bayes bound: Generalization properties of Entropy-SGD and data-dependent priors," in Proceedings of the 35th International Conference on Machine Learning (ICML), 2018, pp. 1377-1386.

[52] S. L. Smith and Q. V. Le, "A Bayesian perspective on generalization and stochastic gradient descent," in International Conference on Learning Representations, 2018.

[53] Y. Zhang, A. M. Saxe, M. S. Advani, and A. A. Lee, "Energy-entropy competition and the effectiveness of stochastic gradient descent in machine learning," Molecular Physics, vol. 116, no. 21-22, pp. 3214-3223, 2018.

[54] I. Nachum and A. Yehudayoff, "Average-case information complexity of learning," in International Conference on Algorithmic Learning Theory (ALT), 2019, pp. 633-646.

[55] R. Livni and S. Moran, "A limitation of the PAC-Bayes framework," in Advances in Neural Information Processing Systems, vol. 33, 2020.

[56] P. Harsha, R. Jain, D. McAllester, and J. Radhakrishnan, "The communication complexity of correlation," IEEE Transactions on Information Theory, vol. 56, no. 1, pp. 438-449, 2009.

[57] M. Havasi, R. Peharz, and J. M. Hernàndez-Lobato, "Minimal random code learning: Getting bits back from compressed model parameters," in International Conference on Learning Representations, 2019.

[58] C. T. Li and A. El Gamal, "Strong functional representation lemma and applications to coding theorems," IEEE Transactions on Information Theory, vol. 64, no. 11, pp. 6967-6978, 2018. 


\section{A The strong functional representation lemma and single-draw bounds}

In this section, we highlight a functional characterization of the mutual information in relation to a single-draw generalization bound of the form, $\operatorname{Pr}(|\mathrm{g}(W, S)|>\epsilon) \leq \delta$, due to [4].

A randomized learning algorithm $P_{W \mid S}$ can be viewed as a noisy channel that maps the input sample $S$ to conditional distributions of hypotheses $W$ in $\mathcal{W}$. Consider the one-shot noisy channel simulation problem [56]. Alice and Bob share a common random string $R$, possibly of unbounded length, generated in advance. Alice observes a sample $s \in \mathcal{Z}^{n}$ drawn according to $P_{S}$ and communicates a prefix-free message $M$ to Bob via a noiseless channel such that Bob can output a hypothesis $w \in \mathcal{W}$ that is distributed according to $P_{W \mid S=s}$. Harsha et al. [56] showed that the minimum expected description length of $M$ (in bits) needed to accomplish this task is roughly equal to the input-output mutual information $I(S ; W)$. Variations on this theme have appeared in a learning-theoretic setting [38], and by way of the bits-back argument due to [49]; see, e.g., [57]. More generally, we note the following functional characterization of the mutual information:

Theorem 23 (Strong functional representation lemma (SFRL) [58]). For any pair of jointly distributed random variables $(S, W)$ with $I(S ; W)<\infty$, there exists a random variable $R$ independent of $S$ such that $W$ can be represented as a deterministic function of $S$ and $R$, and

$$
I(S ; W) \leq H(W \mid R) \leq I(S ; W)+\log (I(S ; W)+1)+4 .
$$

The SFRL implies the existence of a random variable $R \Perp S$ such that $H(W \mid R) \approx I(S ; W)$. In the one-shot channel simulation problem, for instance, $R$ encapsulates the common randomness shared between Alice and Bob.

Consider the case for the $\{0,1\}$-valued loss. If the algorithm $P_{W \mid S}$ is deterministic, then we have $I:=I(S ; W)=H(W)$. By Markov's inequality, with probability of at least $1-\delta$, we have $P_{W}(w) \geq e^{-I / \delta}$. Let $\mathcal{W}_{0} \subseteq \mathcal{W}$ be the set of hypotheses so that $P_{W}(w) \geq e^{-I / \delta}$. The size of $\mathcal{W}_{0}$ is at most $e^{I / \delta}$ since $1=\operatorname{Pr}(\mathcal{W}) \geq \operatorname{Pr}\left(\mathcal{W}_{0}\right)=\sum_{w \in \mathcal{W}_{0}} P_{W}(w) \geq\left|\mathcal{W}_{0}\right| e^{-I / \delta}$. By the Chernoff-Hoeffding bound, for every $w$ in $\mathcal{W}$,

$$
\operatorname{Pr}_{S}(|\mathrm{~g}(w, S)|>\epsilon) \leq 2 e^{-2 n \epsilon^{2}} \quad \forall \epsilon>0 .
$$

Applying the union bound over all $w \in \mathcal{W}_{0}$, the probability of error for the algorithm is $2\left|\mathcal{W}_{0}\right| e^{-2 n \epsilon^{2}}+$ $\delta$, where the second summand is for the case where the algorithm outputs a function outside $\mathcal{W}_{0}$. Hence, for every $w \in \mathcal{W}_{0}$, the empirical risk is close to the true risk for $n=\Omega\left(\frac{I}{\delta \epsilon^{2}}\right)$ with probability of at least $1-\delta$.

Any randomized algorithm can be simulated by randomly sampling a deterministic algorithm from some distribution $R$ before observing the input $S$. By the SFRL, these algorithms have the property that on average (over $R), H(W \mid R) \approx I(S ; W)$. Using the argument for the deterministic case and integrating over $R$, we can bound the probability of error for the randomized case as

$$
\underset{S, W}{\operatorname{Pr}}(|g(W, S)|>\epsilon)=O\left(\frac{I(S ; W)}{n \epsilon^{2}}\right) .
$$

An analogous bound for the sub-Gaussian loss appears in [2, Theorem 3]. 


\section{B Proofs}

\section{B.1 Proofs for Section 3.1}

The following variational characterization of the KL divergence is a rephrasing of Lemma 3:

Lemma 24 (Donsker-Varadhan). Let $P, Q$ be probability measures on $\mathcal{W}$, and let $\mathcal{F}$ denote the set of real-valued measurable functions $f$ on $\mathcal{W}$ such that $\mathbb{E}_{Q}\left[e^{f(W)}\right]<\infty$. If $D(P \| Q)<\infty$, then for every $f \in \mathcal{F}$, we have

$$
D(P \| Q)=\sup _{f \in \mathcal{F}}\left\{\mathbb{E}_{P}[f(W)]-\ln \mathbb{E}_{Q}\left[e^{f(W)}\right]\right\}
$$

where the supremum is attained when $f=\ln \frac{\mathrm{d} P}{\mathrm{~d} Q}$.

We include a proof of the information exponential inequality, since we will use the arguments.

Proof of Lemma 4. Applying the Donsker-Varadhan Lemma 24 to the function,

$$
f(w)=n \beta\left(M_{\beta}(w)-L_{S}(w)\right),
$$

we obtain,

$$
n \beta \mathbb{E}_{P}\left[M_{\beta}(W)-L_{S}(W)\right]-D(P \| Q) \leq \ln \mathbb{E}_{Q}\left[e^{n \beta\left(M_{\beta}(W)-L_{S}(W)\right)}\right] .
$$

Exponentiating both sides of (17) and taking expectations w.r.t. $S \sim \mu^{\otimes n}$, we have

$$
\mathbb{E}_{S} \exp \left\{n \beta \mathbb{E}_{P}\left[M_{\beta}(W)-L_{S}(W)\right]-D(P \| Q)\right\} \leq \mathbb{E}_{S} \mathbb{E}_{Q}\left[e^{n \beta\left(M_{\beta}(W)-L_{S}(W)\right)}\right] .
$$

Since $Z_{i} \stackrel{\text { i.i.d. }}{\sim} \mu$, for any $w \in \mathcal{W}$ and $\beta>0$, we have $e^{-n \beta M_{\beta}(w)}=\mathbb{E}_{S \sim \mu \otimes n}\left[e^{-n \beta L_{S}(w)}\right]$. This observation and Fubini's theorem implies that the right hand side of (18) is equal to one. This proves the IEI.

Proof of Theorem 6. Letting $R(S):=n \beta \mathbb{E}_{P}\left[M_{\beta}(W)-L_{S}(W)\right]-D(P \| Q)$, by Lemma 4, we have $\mathbb{E}_{S}\left[e^{R(S)}\right] \leq 1$. By Markov's inequality,

$$
\operatorname{Pr}_{S}\left(R(S)>\ln \frac{1}{\delta}\right)=\operatorname{Pr}_{S}\left(e^{R(S)}>\frac{1}{\delta}\right) \leq \mathbb{E}_{S}\left[e^{R(S)}\right] \delta \leq \delta .
$$

Therefore, with probability of at least $1-\delta$ over the choice of $S \sim \mu^{\otimes n}$, we have for all $P \ll Q$,

$$
\mathbb{E}_{P}\left[M_{\beta}(W)\right] \leq \mathbb{E}_{P}\left[L_{S}(W)\right]+\frac{1}{n \beta}\left(D(P \| Q)+\ln \frac{1}{\delta}\right)
$$

By assumption (5),

$$
\sup _{w \in \mathcal{W}}\left[L_{\mu}(w)-M_{\beta}(w)\right] \leq \frac{\psi(\beta)}{\beta}, \forall \beta>0,
$$

when (6) follows. 
For the bound in expectation, note that by Jensen's inequality, $e^{\mathbb{E}_{S}[R(S)]} \leq \mathbb{E}_{S}\left[e^{R(S)}\right] \leq 1$, which implies $\mathbb{E}_{S}[R(S)] \leq 0$, when we have

$$
\mathbb{E}_{S W}\left[M_{\beta}(W)\right] \leq \mathbb{E}_{S W}\left[L_{S}(W)\right]+\frac{1}{n \beta} D\left(P \| Q \mid P_{S}\right) .
$$

Using (5), and rearranging and optimizing, we have

$$
\begin{aligned}
\mathbb{E}_{S W}[\mathrm{~g}(W, S)] & \leq \inf _{\beta>0} \frac{\frac{1}{n} D\left(P \| Q \mid P_{S}\right)+\psi(\beta)}{\beta} \\
& =\psi^{*-1}\left(\frac{D\left(P \| Q \mid P_{S}\right)}{n}\right),
\end{aligned}
$$

where the second equality follows from Lemma 2.

The proof of Proposition 10 follows that of [23, Lemma 8], extending it to sub-Gaussian losses. Proof of Proposition 10. For $0<u<v$, and $i=0, \ldots,\left\lceil\log _{\alpha} \frac{v}{u}\right\rceil-1$, for all $i$ let $\beta_{i}=u \alpha^{i}$ be selected before the draw of the training sample. Then for every $\beta \in[u, v]$, there is a $\beta_{i}$ such that $\beta_{i} \leq \beta \leq \alpha \beta_{i}$.

We can extend (3) by applying a union bound over the $\beta_{i}$ 's, so that for all $P$ with probability of at least $1-\delta$ over the draw of $S$, the following holds simultaneously for all $\beta_{i}$ :

$$
\mathbb{E}_{P}\left[M_{\beta_{i}}(W)\right] \leq \mathbb{E}_{P}\left[L_{S}(W)\right]+\frac{\alpha}{n \beta_{i}}\left(D(P \| Q)+\ln \frac{\left\lceil\log _{\alpha} \frac{v}{u}\right\rceil}{\delta}\right) .
$$

By Proposition 1 item 2), for any $w \in \mathcal{W}, M_{\beta}(w)$ is a nonincreasing of $\beta$. Thus for any $\beta \in[u, v]$ and $\beta_{i}$ such that $\beta_{i} \leq \beta \leq \alpha \beta_{i}, M_{\beta}(w) \leq M_{\beta_{i}}(w)$ and $\frac{1}{\beta_{i}} \leq \frac{\alpha}{\beta}$. Moreover, since $\ell(w, Z)$ is $\sigma$-subGaussian under $\mu$ by assumption, we have for all $w \in \mathcal{W}$ and $\beta>0, L_{\mu}(w) \leq M_{\beta}(w)+\frac{\beta}{2} \sigma^{2}$. Hence, with probability of at least $1-\delta$ we have,

$$
\mathbb{E}_{P}\left[L_{\mu}(W)\right] \leq \mathbb{E}_{P}\left[L_{S}(W)\right]+\frac{\alpha}{n \beta}\left(D(P \| Q)+\ln \frac{\left\lceil\log _{\alpha} \frac{v}{u}\right\rceil}{\delta}\right)+\frac{\beta \sigma^{2}}{2}
$$

Letting $J=D(P \| Q)+\ln \frac{\log _{\alpha} \sqrt{n}+K}{\delta}$, we find that the value for $\beta$ that optimizes the right hand side of the bound in the statement of the proposition is bounded from below by $\sqrt{\frac{2 \alpha}{n \sigma^{2}}}$. Letting $u=\frac{1}{\sqrt{n}} \min \left\{\sqrt{\frac{2 \alpha}{\sigma^{2}}}, v\right\}$ and plugging it in (20) completes the proof.

\section{B.2 Proofs for Section 3.2}

Proof of Proposition 12. Applying the Donsker-Varadhan lemma 24 to the function, $f(w)=n \beta \mathrm{g}(w, \tilde{Z}, U)$, and following the same steps as in the proof of Lemma 4, we arrive at

$$
\mathbb{E}_{\tilde{Z}, U} \exp \left\{n \beta \mathbb{E}_{P}[\mathrm{~g}(W, \tilde{Z}, U)]-D(P \| Q)\right\} \leq \mathbb{E}_{Q} \mathbb{E}_{\tilde{Z}, U}\left[e^{n \beta \mathrm{g}(W, \tilde{Z}, U)}\right]=\mathbb{E}_{Q} \mathbb{E}_{\tilde{Z}} \mathbb{E}_{U}\left[e^{n \beta \mathrm{g}(W, \tilde{Z}, U)}\right],
$$

where the last equality follows since $\tilde{Z} \Perp U$. Since $\ell \in[0,1], \mathrm{g}(W, \tilde{Z}, U)$ is $\frac{1}{\sqrt{n}}$-sub-Gaussian. Morever, $\mathbb{E}_{U}[\mathrm{~g}(W, \tilde{Z}, U)]=0$. By Hoeffding's lemma, we have $\mathbb{E}_{\tilde{Z}} \mathbb{E}_{U}\left[e^{n \beta \mathrm{g}(W, \tilde{Z}, U)}\right] \leq e^{n \beta^{2} / 2}$, and hence

$$
\mathbb{E}_{\tilde{Z}, U} \exp \left\{n \beta \mathbb{E}_{P}[\mathrm{~g}(W, \tilde{Z}, U)]-D(P \| Q)-\frac{n \beta^{2}}{2}\right\} \leq 1
$$


(8) then follows by an application of Markov's inequality.

Let $R(\tilde{Z}, U)=n \beta \mathbb{E}_{P}[\mathrm{~g}(W, \tilde{Z}, U)]-D(P \| Q)-\frac{n \beta^{2}}{2}$. From (22), and using Jensen's inequality, we have $e^{\mathbb{E}_{\tilde{Z}, U}[R(\tilde{Z}, U)]} \leq \mathbb{E}_{\tilde{Z}, U}\left[e^{R(\tilde{Z}, U)}\right] \leq 1$, which implies

$$
\mathbb{E}_{\tilde{Z}, U, W}[\mathrm{~g}(W, \tilde{Z}, U)] \leq \inf _{\beta>0}\left(\frac{D\left(P_{W \mid \tilde{Z} U} \| Q_{W \mid \tilde{Z}} \mid P_{\tilde{Z} U}\right)}{n \beta}+\frac{\beta}{2}\right)=\sqrt{\frac{2 \cdot D\left(P_{W \mid \tilde{Z} U} \| Q_{W \mid \tilde{Z}} \mid P_{\tilde{Z} U}\right)}{n}}
$$

and we have shown (9).

Under the oracle prior $Q_{W \mid \tilde{Z}}=P_{W \mid \tilde{Z}}$, we have $D\left(P_{W \mid \tilde{Z} U} \| P_{W \mid \tilde{Z}} \mid P_{\tilde{Z} U}\right)=I(W ; U \mid \tilde{Z})$. By noting that $\mathbb{E}_{\tilde{Z}, U, W}[\mathrm{~g}(W, \tilde{Z}, U)]=\mathbb{E}_{\tilde{Z}, U, W}\left[\mathrm{~g}\left(W, \tilde{Z}_{U}\right)\right]$, we recover $[7$, Theorem 2(1)].

\section{B.3 Omitted details in Section 3.3}

We show how inequality (3) relates to other well-known PAC-Bayesian inequalities such as the "PAC-Bayes-KL-inequality" [22,35]. Applying the Donsker-Varadhan lemma to the function $f(w)=$ $n \beta\left(L_{\mu}(w)-L_{S}(w)\right)$, which involves the true risk $L_{\mu}(w)$ instead of the annealed expectation $M_{\beta}(w)$ (see 16), and following the same steps as in the proof of (3) in Theorem 5, we arrive at the following PAC-Bayesian bound:

$$
\begin{aligned}
\operatorname{Pr}_{S \sim \mu^{\otimes n}}\left(\mathbb{E}_{P}\left[L_{\mu}(W)\right] \leq \mathbb{E}_{P}\left[L_{S}(W)\right]+\frac{1}{n \beta}[\right. & D(P \| Q)+\ln \frac{1}{\delta} \\
& +\ln \mathbb{E}_{Q} \mathbb{E}_{S^{\prime} \sim \mu}^{\otimes n} e^{\left.\left.n \beta\left(L_{\mu}(W)-L_{S^{\prime}}(W)\right)\right]\right) \geq 1-\delta .}
\end{aligned}
$$

For an explicit comparison of (23) with (3), we write the latter as

$$
\begin{aligned}
\operatorname{Pr}_{S \sim \mu^{\otimes n}}\left(\mathbb{E}_{P}\left[M_{\beta}(W)\right] \leq \mathbb{E}_{P}\left[L_{S}(W)\right]+\frac{1}{n \beta}[\right. & D(P \| Q)+\ln \frac{1}{\delta} \\
& +\underbrace{\left.\left.\ln \mathbb{E}_{Q} \mathbb{E}_{S^{\prime} \sim \mu \otimes n} e^{n \beta\left(M_{\beta}(W)-L_{S^{\prime}}(W)\right)}\right]\right) \geq 1-\delta,}_{=0}
\end{aligned}
$$

where the last term in the right hand side of the bound in (24) vanishes since

$$
e^{-n \beta M_{\beta}(w)}=\mathbb{E}_{S^{\prime} \sim \mu^{\otimes n}}\left[e^{-n \beta L_{S^{\prime}}(w)}\right]
$$

for any $w \in \mathcal{W}$ and $\beta>0$. In contrast, the term $\ln \mathbb{E}_{Q} \mathbb{E}_{S^{\prime} \sim \mu}^{\otimes n} e^{n \beta\left(L_{\mu}(W)-L_{S^{\prime}}(W)\right)}$ involving the true risk in (23) is, in general, positive.

Specializing to the case of a $\{0,1\}$-valued loss, fix $\beta=1$, and let $\Delta:[0,1] \times[0,1] \rightarrow \mathbb{R}$ be a convex function. Applying the Donsker-Varadhan lemma to the function, $f(w)=n \Delta\left(L_{S}(w), L_{\mu}(w)\right)$, following the same steps as in the proof of (3) in Theorem 5, and by noting that

$$
\left.\Delta\left(\mathbb{E}_{P}\left[L_{S}(W)\right], \mathbb{E}_{P}\left[L_{\mu}(W)\right]\right)\right) \leq \mathbb{E}_{P}\left[\Delta\left(L_{S}(W), L_{\mu}(W)\right)\right],
$$


we arrive at the following PAC-Bayesian bound (see, e.g., [22, Lemma 3], [26, Theorem 2.1], [29, Equation 4]):

$$
\begin{aligned}
\operatorname{Pr}_{S \sim \mu^{\otimes n}}\left(\Delta\left(\mathbb{E}_{P}\left[L_{S}(W)\right], \mathbb{E}_{P}\left[L_{\mu}(W)\right]\right)\right) \leq \frac{1}{n}[ & D(P \| Q)+\ln \frac{1}{\delta} \\
& +\ln \mathbb{E}_{Q} \mathbb{E}_{S^{\prime} \sim \mu^{\otimes n}} e^{\left.\left.n \Delta\left(L_{S^{\prime}}(W), L_{\mu}(W)\right)\right]\right) \geq 1-\delta .}
\end{aligned}
$$

For $x, y \in[0,1]$, the binary KL divergence is $\mathrm{kl}(y \| x)=y \ln \frac{y}{x}+(1-y) \ln \frac{1-y}{1-x}$. The PAC-Bayes-KLinequality $[22,35]$ comes about by upper-bounding the log-exponential-moment term involving the true risk in the right hand side of the bound in (25): For $\Delta(y, x)=\mathrm{kl}(y \| x)$, Maurer [22] showed that for $n \geq 8, \mathbb{E}_{Q} \mathbb{E}_{S^{\prime}}\left[e^{n \Delta\left(L_{S^{\prime}}(W), L_{\mu}(W)\right)}\right] \leq 2 \sqrt{n}$, when we have

$$
\left.\operatorname{Pr}_{S \sim \mu^{\otimes n}}\left(\operatorname{kl}\left(\mathbb{E}_{P}\left[L_{S}(W)\right], \mathbb{E}_{P}\left[L_{\mu}(W)\right]\right)\right) \leq \frac{1}{n}\left[D(P \| Q)+\ln \frac{2 \sqrt{n}}{\delta}\right]\right) \geq 1-\delta .
$$

(26) can be interpreted as a "non-parametric" version of McAllester's linear PAC-Bayes bound (11) that is uniform in $\beta$ at the cost of a $O\left(\frac{\ln \sqrt{n}}{n}\right)$ term.

Letting $\Delta(y, x)=2(y-x)^{2}$ in (25) leads to the bound in [17], while letting $\Delta(y, x)=(y-x)^{2} /(2 x)$ leads to that in [28].

Under a sub-gamma loss assumption, the bounds in either (23) or (24) lead to (see Corollary 9):

$$
\operatorname{Pr}_{S \sim \mu \otimes n}\left(\mathbb{E}_{P}\left[L_{\mu}(W)\right] \leq \mathbb{E}_{P}\left[L_{S}(W)\right]+\frac{1}{n}\left[D(P \| Q)+\ln \frac{1}{\delta}\right]+\frac{\sigma^{2}}{2(1-c)}\right) \geq 1-\delta .
$$

\section{B.4 Proofs for Section 4}

The proof of Proposition 19 follows closely that of [41, Theorem 4.2].

Proof of Proposition 19. For every $Q \in \mathcal{M}(\mathcal{W})$, let

$$
F(Q)=\left\{S^{\prime} \in \mathcal{Z}^{n}: \exists P \in \mathcal{M}(\mathcal{W}), \mathbb{E}_{P}\left[M_{\beta}(W)\right] \geq \mathbb{E}_{P}\left[L_{S^{\prime}}(W)\right]+\frac{1}{n \beta}\left(D(P \| Q)+\ln \frac{1}{\delta^{\prime}}\right)\right\} .
$$

By Theorem 5, we have $\operatorname{Pr}_{S^{\prime} \sim \mu^{\otimes n}}\left(S^{\prime} \in F(Q)\right) \leq \delta^{\prime}$. From (12), we have

$$
\operatorname{Pr}_{S \sim \mu^{\otimes n}}\left(S \in F\left(Q^{0}(S)\right)\right) \leq e^{I_{\infty, \mu}^{\alpha}\left(Q^{0}, n\right)} \cdot \operatorname{Pr}_{\left(S, S^{\prime}\right) \sim \mu^{\otimes 2 n}}\left(S^{\prime} \in F\left(Q^{0}(S)\right)\right)+\alpha \leq e^{I_{\infty, \mu}^{\alpha}\left(Q^{0}, n\right)} \cdot \delta^{\prime}+\alpha .
$$

Letting $\delta:=e^{I_{\infty, \mu}^{\alpha}\left(Q^{0}, n\right)} \cdot \delta^{\prime}+\alpha$, for $\alpha \in(0, \delta)$ we have,

$$
\begin{aligned}
\operatorname{Pr}_{S \sim \mu^{\otimes n}}\left(\exists P \in \mathcal{M}(\mathcal{W}), \mathbb{E}_{P}\left[M_{\beta}(W)\right]\right. & \geq \mathbb{E}_{P}\left[L_{S}(W)\right] \\
& \left.+\frac{1}{n \beta}\left(D\left(P \| Q^{0}(S)\right)+\ln \frac{1}{\delta-\alpha}+I_{\infty, \mu}^{\alpha}\left(Q^{0}, n\right)\right)\right) \leq \delta .
\end{aligned}
$$

The proof is complete by replacing $I_{\infty, \mu}^{\alpha}\left(Q^{0}, n\right)$ with the bound in Theorem 17 , and choosing $\alpha=$ $\frac{\delta}{2}$. 


\section{B.5 Proofs for Section 5.1}

We show how to optimize the bound in Corollary 13 w.r.t. the parameters $\beta$ and $\lambda$.

First, note that the bound in Corollary 13 holds uniformly for all $\beta>1$ at an additional cost arising from a union bound argument [21, Theorem 1.2.7]: For $\alpha>1$,

$$
\mathbb{E}_{P}\left[L_{\mu}\left(f_{W}\right)\right] \leq \inf _{\beta>1} \Phi_{\beta}^{-1}\left\{\mathbb{E}_{P}\left[L_{S}\left(f_{W}\right)\right]+\frac{\alpha}{n \beta}\left[D(P \| Q)+\ln \frac{1}{\delta}+2 \ln \left(\frac{\ln \alpha^{2} \beta n}{\ln \alpha}\right)\right]\right\} .
$$

Second, we select $\lambda$ before the draw of the training sample from a finite grid of possible values: Following [31,48], let $\lambda=c e^{-j / b}$ for some $j \in \mathbb{N}$ and fixed $b \in \mathbb{N}, c \in(0,1)$, where $b$ and $c$ control, resp., the resolution and size of the grid. If (28) holds for each $j \in \mathbb{N}$ with probability of at least $1-\frac{6 \delta}{\pi^{2} j^{2}}$, then by the union bound, it holds for all $j \in \mathbb{N}$ simultaneously with probability of at least $1-\delta$, since $\sum_{j=1}^{\infty} \frac{6}{\pi^{2} j^{2}}=1$. Solving for $j$ in terms of $\lambda$, we have

$$
\begin{aligned}
\mathbb{E}_{P}\left[L_{\mu}\left(f_{W}\right)\right] \leq \inf _{\beta>1, \lambda \in(0, c)} \Phi_{\beta}^{-1}\{ & \mathbb{E}_{P}\left[L_{S}\left(f_{W}\right)\right] \\
& \left.+\frac{\alpha}{n \beta}\left[D(P \| Q)+\ln \left(\frac{\pi^{2} b^{2}}{6 \delta}\left(\ln \frac{c}{\lambda}\right)^{2}\right)+2 \ln \left(\frac{\ln \alpha^{2} \beta n}{\ln \alpha}\right)\right]\right\} .
\end{aligned}
$$

Finally, we account for the cost of using a Monte Carlo estimate of the empirical risk, $\hat{L}_{S}\left(f_{W}\right)=$ $\frac{1}{m} \sum_{i=1}^{m} L_{S}\left(f_{W_{i}}\right)$, where $W_{i} \stackrel{\text { i.i.d. }}{\sim} P$. By an application of the Chernoff bound [48, Theorem 2.5] and Pinsker's inequality, for any $\delta^{\prime} \in(0,1)$, we have with probability of at least $1-\delta^{\prime}, \mathbb{E}_{P}\left[L_{S}\left(f_{W}\right)\right] \leq$ $\hat{L}_{S}\left(f_{W}\right)+\sqrt{\frac{1}{2 m} \ln \frac{2}{\delta^{\prime}}}$.

By another application of the union bound, Corollary 13 finally takes the form: For any $\delta, \delta^{\prime} \in$ $(0,1)$, fixed $\alpha>1, c \in(0,1), b \in \mathbb{N}$, and $m, n \in \mathbb{N}$, with probability of at least $1-\delta-\delta^{\prime}$ over a draw of $S \sim \mu^{\otimes n}$ and $W \sim(P)^{\otimes m}$,

$$
\mathbb{E}_{P}\left[L_{\mu}\left(f_{W}\right)\right] \leq \inf _{P \in \mathcal{G}, \beta>1, \lambda \in(0, c)} \Phi_{\beta}^{-1}\left\{\hat{L}_{S}\left(f_{W}\right)+\frac{\alpha}{n \beta} D(P \| Q)+R\left(\lambda, \beta ; \delta, \delta^{\prime}\right)\right\}
$$

where $R=\frac{2 \alpha}{n \beta} \ln \left[\frac{\ln \alpha^{2} \beta n}{\ln \alpha}\right]+\frac{\alpha}{n \beta} \ln \left[\frac{\pi^{2} b^{2}}{6 \delta}\left(\ln \frac{c}{\lambda}\right)^{2}\right]+\sqrt{\frac{1}{2 m} \ln \frac{2}{\delta^{\prime}}}$.

\section{B.6 Proofs for Section 5.3}

Proof of Lemma 21. Letting $\theta=w-w_{P}$, and $P^{\prime}=P-w_{P}$, note that $\theta^{\top} H \theta=\operatorname{Tr}\left(\theta^{\top} H \theta\right)=$ $\operatorname{Tr}\left(H \theta \theta^{\top}\right)$. Hence

$$
\mathbb{E}_{P^{\prime}}\left[\frac{1}{2} \theta^{\top} H \theta\right]=\mathbb{E}_{P^{\prime}}\left[\frac{1}{2} \operatorname{Tr}\left(H \theta \theta^{\top}\right)\right]=\frac{1}{2} \operatorname{Tr}\left(H \mathbb{E}_{P^{\prime}}\left[\theta \theta^{\top}\right]\right)=\frac{1}{2} \operatorname{Tr}\left(H \Sigma_{P}\right) .
$$

For $Q \sim \mathcal{N}\left(w_{Q}, \Sigma_{Q}\right)$ and $P \sim \mathcal{N}\left(w_{P}, \Sigma_{P}\right)$, we have

$$
\begin{aligned}
\mathbb{E}_{P^{\prime}} & {\left[\frac{1}{2} \theta^{\top} H \theta\right]+(n \beta)^{-1} D(P \| Q) } \\
& =\frac{1}{2} \operatorname{Tr}\left(H \Sigma_{P}\right)+(n \beta)^{-1} D(P \| Q) \\
& =\frac{\operatorname{Tr}\left(H \Sigma_{P}\right)}{2}+\frac{(n \beta)^{-1}}{2}\left(\ln \frac{\operatorname{det} \Sigma_{Q}}{\operatorname{det} \Sigma_{P}}+\operatorname{Tr}\left(\Sigma_{Q}^{-1} \Sigma_{P}\right)-k+\left(w_{Q}-w_{P}\right)^{\top} \Sigma_{Q}^{-1}\left(w_{Q}-w_{P}\right)\right) .
\end{aligned}
$$


The derivative of the RHS w.r.t. $\Sigma_{P}$ is $\frac{1}{2}\left[H-(n \beta)^{-1} \Sigma_{P}^{-1}+(n \beta)^{-1} \Sigma_{Q}^{-1}\right]^{\top}$, where we have used the fact that $\nabla_{A} \operatorname{Tr}(A B)=B^{\top}$, and $\nabla_{A} \ln \operatorname{det}(A)=\left(A^{-1}\right)^{\top}$. Setting the derivative to zero and $\Sigma_{Q}=\lambda^{-1} I_{k}$ yields the result.

Proof of Proposition 22. The proof follows from Theorem 5, and the fact that for $Q=\mathcal{N}\left(w_{Q}, \lambda^{-1} I_{k}\right)$, $P=\mathcal{N}\left(w_{P}, H_{\lambda}^{-1}\right)$ such that $\lambda_{i} \geq \lambda>0$ for all $i$, we have

$$
D(P \| Q)=\frac{1}{2}\left(\lambda\left\|w_{Q}-w_{P}\right\|^{2}+\sum_{i=1}^{k} \ln \frac{\lambda_{i}}{\lambda}+\sum_{i=1}^{k}\left(\frac{\lambda}{\lambda_{i}}-1\right)\right) \leq \frac{1}{2}\left(\lambda\left\|w_{Q}-w_{P}\right\|^{2}+\sum_{i=1}^{k} \ln \frac{\lambda_{i}}{\lambda}\right) .
$$

